\title{
Can quantum theory be understood?
}

\author{
Physics has a more urgent responsibility than it appreciates to make the most general tool of its trade more widely \\ understood, to which end proofs positive are more relevant than abstractions.
}

SOME years ago, a group of able physics teachers met to plan a new curriculum for the sixth forms of British schools and were asked, "What will you put in about quantum mechanics?" To a man and woman they shook their heads, saying that they did not know, but that they were prepared to follow whatever instructions they were given. In the event, the curriculum turned out to be more adventurous than many feared, but the willingness of normally determined people to follow guidance about quantum mechanics is a telling proof of how poorly the matter is generally understood.

So much is easily verified by asking artlessly at dinner parties and the like some variant of the question "What does quantum mechanics mean to you?" With luck, the response may be the opinion that quantum theory implies that nothing is as certain as Newton said or, with some brave extrapolation, that nothing at all is certain.

A modicum of technical education, on the other hand, usually yields one or both of two elaborations of this doctrine. One is a reference to the Uncertainty Principle and to the limitations that it imposes on the accuracy of physical measurements. Another is the doctrine that "energy is quantized", offered as an explanation why the electronic states of atoms and other structures, such as metals, can accommodate electrons only in states whose energy is predetermined and, often, precalculable.

The trouble with even these statements, their imprecision apart, is that they are too abstract to carry general conviction. Worse, they seem logically unconnected with each other. Why, for example, should the Uncertainty Principle, which would seem to make quantities fuzzy, be linked with the apparently contradictory notion that the energy of an electron in, say, the ground state of a hydrogen atom is exquisitely exact? Is quantum mechanics about uncertainty or the opposite?

It hardly helps the general understanding to remark that the frequency span of a spectral line is partly a measure of the lifetime of the more energetic state involved, and that time and energy are linked as if they were any other pair of conjugate variables in classical mechanics. What on Earth, the question will be, are they? It will seem irrelevant that the concept, due to William Hamilton, long antedates Einstein and Bohr.

What quantum mechanics, and perhaps the reputation the rest of physics, needs is a series of phenomena that will stick in the NATURE · VOL 361 · 11 FEBRUARY 1993 general mind and serve as repeated reminders of what the field has to say for itself. That, after all, is why biology enjoys such a good press. From infection to digestion to reproduction, everybody has in mind firsthand experience of body as machine. Physics, and the quantum mechanics part of it especially, is necessarily more artificial, requiring experiments of some kind. But that impediment is not insurmountable.

There is, for example, the question of the production of electron-pairs. The world is full of photographs (some going back to before the discovery of the positron) taken in cloud chambers and other devices placed in magnetic fields that display the elegantly backward curving tracks of an electron and a positron apparently diverging from a point in space on which nothing else impinges. The standard tale is that there is instead a photon, invisible because it has no electric charge, which has created an electron pair from nothing. The response invited is something along the lines of "Isn't that astonishing!" The impression is reinforced that physics is either akin to magic or that it seeks to be.

Why not tell it like it is? The Uncertainty Principle is a good starting point. That empty space in which the electron-pair appears cannot be exactly empty, right, because no entity can be as exactly defined? And in reality, any patch of vacuum is potentially a source of any substantial particle you may specify (except, it seems, the $17-\mathrm{keV}$ neutrino and, for the time being, the top quark)?

So what more natural than that a photon traversing such a patch of space, especially one disturbed by the presence of an atomic nucleus (necessary on kinematic grounds) should exchange its energy for that of an electron-pair? The crucial element in this pedagogical exercise is to plead that the Uncertainty Principle ensures that there is qualitative as well as quantitative uncertainty, but that is simply to tell it like it is.

Sadly, when physics is taught to people not intending to be physicists, the moral usually drawn from the picture of the electron-pair is more often that it illustrates again Einstein's old saw that $E=m c^{2}$, which is proper but unadventurous. Why not use the electron pair as a text for a sermon about the fluctuations of the vacuum, the idea that nothing is potentially the source of everything? That might stick in the general mind.

Casual flicking through physics journals will then provide endless ammunition. If the vacuum is a potential source of electron- pairs, so too is it potentially a source of photons. The frequency of the radiation emitted by a circulating beam of electrons in a synchrotron can be calculated from the work of Maxwell and Lorentz, but the energetic photons that biologists use for $\mathrm{X}$-ray studies of protein crystals have been physically conjured out of the vacuum by nothing more substantial than the curvature of the electric current represented by the circulating electrons (which in this case are not destroyed).

Here, for what it is worth, is the neatest experiment yet carried out to demonstrate that the vacuum is potentially an endless source of photons, even when their effects are never manifested. A group at Yale University (Sukenik, C.I., et al. Phys. Rev. Lett. 70, 560; 1 February 1993) has constructed a cavity consisting of the wedge-shaped region between two gold-covered surfaces flat to within 3 nanometres. The opening of the wedge is determined by sliding a nickel foil $1.2 \mu \mathrm{m}$ thick between the flats.

In such a cavity, only some frequencies of the radiation field can be supported, as they say in the computer business. So what happens if you shoot a beam of ordinary unexcited sodium atoms through the wedgeshaped space? Because the wedge does not allow many of the radiation frequencies found in larger patches of vacuum, the energy of the electrons in the sodium is shifted from what it would ordinarily be. That, at least, is the prediction. What matters is that it appears to have been confirmed by a measurement of the transmission of a carefully tuned laser beam through the emergent beam of sodium atoms. The experiment has not merely demonstrated that the vacuum is not devoid of substance, but that the consequences are apparent even when there is no magical appearance of something unexpected.

To be fair to Sukenik and his colleagues, they are not simply concerned to show that quantum mechanics has substance, but to demonstrate the reality of what is called the Casimir-Polder force the attractive force between two nearby conducting plates occasioned simply by the absence in the intervening cavity of radiation frequencies that cannot, because of the geometry, exist. The force has previously been measured directly, as has been the increased lifetime of an excited atom trapped in such a space. These phenoma, which are artificial, may be a long way from everyday experience, but they are proof positive, not abstractions. 